\title{
Curcumin reverses the sunitinib resistance in clear cell renal cell carcinoma (ccRCC) through the induction of ferroptosis via the ADAMTS18 gene
}

\author{
Ben Xu", Wei-Jie Zhu", Yi-Ji Peng, Si-Da Cheng \\ Department of Urology, Peking University First Hospital and Institute of Urology, Peking University, National Urological Cancer Center, Beijing, \\ China \\ Contributions: (I) Conception and design: B Xu; (II) Administrative support: B Xu; (III) Provision of study materials or patients: YJ Peng; (IV) \\ Collection and assembly of data: SD Cheng; (V) Data analysis and interpretation: WJ Zhu; (VI) Manuscript writing: All authors; (VII) Final approval \\ of manuscript: All authors. \\ \#These authors contributed equally to this work. \\ Correspondence to: Ben Xu. Department of Urology, Peking University First Hospital and Institute of Urology, Peking University, National Urological \\ Cancer Center, 8 Xishiku Street, Xicheng District, Beijing 100034, China. Email: xuben_pku@sina.com.
}

Background: To explore the possible mechanism by which curcumin reverses the sunitinib resistance in clear cell renal cell carcinoma (ccRCC).

Methods: A sunitinib-resistant ccRCC cell model was established. The MTT assay was used to determine the half maximal inhibitory concentration $\left(\mathrm{IC}_{50}\right)$ and drug resistance $(\mathrm{DR})$ index. The effects of curcumin plus sunitinib or sunitinib alone on drug-resistant cell lines were verified by the cell counting kit-8 (CCK-8) assay, colony formation assay, and apoptosis assay. The concentration of iron ions in the cell lines was analyzed using an Abcam Iron Assay Kit. The expressions of ADAMTS18 gene and ferroptosis-related proteins (NCOA4, FTH1 and p53) after curcumin plus sunitinib treatment were analyzed by reverse transcription polymerase chain reaction (RT-PCR) and Western blotting. After transfection of curcumin plus sunitinib/sunitinib alone-treated drug-resistant cell lines with si-ADAMTS18, cell proliferation activity was assessed by the CCK-8 assay, and the protein expression levels of ADAMTS18, NCOA1, FTH1 and $p 53$ were analyzed by Western blotting. After treatment with ferroptosis-1 (Fer-1; a ferroptosis inhibitor), the cell proliferation activity of drug-resistant cell lines treated with curcumin plus sunitinib/sunitinib alone was reassessed using the CCK-8 assay.

Results: Curcumin plus sunitinib inhibited the proliferation of sunitinib-resistant ccRCC cells $(\mathrm{P}<0.05)$. Curcumin significantly decreased the concentration of iron ions and increased the expression of ADAMTS18 gene, while significantly inhibited ferroptosis-related protein expression $(\mathrm{P}<0.05)$. After silencing the ADAMTS18 gene, there was no significant difference in cell proliferation or ferroptosis-related protein expression between curcumin plus sunitinib and sunitinib-treated drug-resistant cell lines $(\mathrm{P}>0.05)$. Ferroptosis inhibitors reversed the inhibitory effect of curcumin on sunitinib-resistant ccRCC cell lines.

Conclusions: Curcumin can reverse the sunitinib resistance in ccRCC, possibly by upregulating the expression of the ADAMTS18 gene to induce ferroptosis.

Keywords: Curcumin; clear cell renal cell carcinoma (ccRCC); ferroptosis; ADAMTS18; sunitinib resistance

Submitted Feb 03, 2021. Accepted for publication Jun 25, 2021.

doi: $10.21037 /$ tcr-21-227

View this article at: https://dx.doi.org/10.21037/tcr-21-227 


\section{Introduction}

Clear cell renal cell carcinoma (ccRCC) is the most common renal malignant tumor and one of the most invasive urinary system cancers (1). Patients with ccRCC are often at an advanced stage when they are first diagnosed, and the prognosis is poor. The tyrosine kinase inhibitor of sunitinib inhibits the oxygen and nutrient supply of tumors by blocking angiogenesis. Although sunitinib can prolong the progression-free survival of patients, drug resistance (DR) easily develops during the treatment process, an issue that must be addressed for the successful treatment of advanced ccRCC (2).

Curcumin is the active ingredient of the perennial herb turmeric (Curcuma longa), which has long been used as a traditional medicine in China, India and other countries. Modern science and technology have deeply explored this ancient remedy, and it has been widely used as an antiinflammatory, antioxidative, and chemopreventative agent for tumor prevention and treatment (3).

Ferroptosis is iron-catalyzed programmed cell death, which is characterized by iron-catalyzed peroxidation of phospholipids and polyunsaturated fatty acids. This phospholipid peroxidation causes cell toxicity, which can be neutralized by iron chelators and lipophilic free radical scavengers, indicating that ferroptosis induces cytotoxicity via lipid peroxidation (4). Studies have shown that iron in cells is a central factor in cancer progression, which explain why antitumor drugs can specifically affect tumor cells instead of normal cells with low iron content (5). It has been proposed that the expression of iron-related regulatory genes in ccRCC cells is significantly increased (6). In addition, compared with that in healthy tissues, the expression of NCOA1 was significantly increased in tumor tissues, which was associated with poorer survival outcomes (7). Mancias et al. proposed that NCOA1 is closely related to ferroptosis mechanism in tumor cells (8).

In 2015, our team first identified that abnormal methylation of the ADAMTS18 gene existed in ccRCC (9). In 2021, our team confirmed that the ADAMTS18 gene could participate in the occurrence and development process of ccRCC and that curcumin, as a botanical drug, can inhibit this process by demethylating the ADAMTS18 gene $(10,11)$. Based on the above findings, this study aimed to explore whether curcumin could affect sunitinib resistance in ccRCC cells through the regulation of the ADAMTS18 gene. We present the following article in accordance with the MDAR reporting checklist (available at https://dx.doi. org/10.21037/tcr-21-227).

\section{Methods}

\section{Drugs and reagents}

Sunitinib and curcumin were purchased from Shanghai PureOne Biotechnology Co., Ltd. (purity $\geq 98 \%$ ), and the stock solutions $(150 \mathrm{mmol} / \mathrm{L})$ were prepared using DMSO and stored at room temperature. DMEM, fetal bovine serum (FBS), CCK-8 reagent, and reverse transcription polymerase chain reaction (RT-PCR) kits were purchased from Thermo Fisher Scientific.

\section{Cell culture}

ccRCC cell lines (A498 and 786-O) were purchased from Medical Discovery Leader (Beijing, China) and cultured in medium containing 10\% ultracentrifuged FBS (Invitrogen, CA, USA), $1 \mathrm{mM}$ sodium pyruvate (Invitrogen), penicillin (100 U/mL; Invitrogen), and streptomycin $(10 \mathrm{mg} / \mathrm{mL}$; Invitrogen) at $37{ }^{\circ} \mathrm{C}$ in a humidified environment with $5 \%$ $\mathrm{CO}_{2}$. The cell culture also contained $0.05 \%$ trypsin and $0.02 \%$ ethylenediaminetetraacetic acid (HyClone).

\section{Establishment of sunitinib-resistant cell lines}

A498 and 786-O cells were treated with sunitinib at an initial concentration of $0.5 \mu \mathrm{M}$, and the cell culture medium was replaced. After the cells were stabilized, the concentration of sunitinib was increased stepwise by $0.5 \mu \mathrm{M}$ to gradually establish sunitinib-resistant (15 $\mu \mathrm{M}$ sunitinib) A498 and 786-O cells.

\section{MTT assay}

The cell concentration was adjusted to inoculate $100 \mu \mathrm{L}$ per well into 96-well plates. After cells were adhered to the plates, serial dilution of sunitinib were added. Five replicate wells were used for each concentration as well as a blank control. After $48 \mathrm{~h}$ of culture, $20 \mu \mathrm{L}$ of MTT solution $(5 \mathrm{mg} / \mathrm{mL})$ was added to each well. After $4 \mathrm{~h}$ of culture at $37^{\circ} \mathrm{C}, 150 \mu \mathrm{L}$ of DMSO was added to each well. Plates were shaken at low speed for $10 \mathrm{~min}$ to fully dissolve the purple crystals, and the absorbance was measured at $570 \mathrm{~nm}$ using a microplate reader. Cell inhibition rate $(\mathrm{IC})=[1-$ optical density (OD) value of the experimental group/OD value of the control group], and DR index = half maximal inhibitory 
Table 1 The primers of $A D A M T S 18, N C O A, F T H 1, p 53$ and GAPDH for RT-PCR

\begin{tabular}{lll}
\hline Genes & F 5'-3' & R 5'-3' \\
\hline NCOA4 & GAGGTGTAGTGATGCACGGAG & GACGGCTTATGCAACTGTGAA \\
FTH1 & GGTGGCCGAATCTTCCTTCA & GCCAGTTTGTGCAGTTCCAG \\
ADAMTS18 & CGCCAGCGGATTAAATGATGA & GAAAATCGGTAGTGCAGGGAG \\
p53 & CAGCACATGACGGAGGTTGT & TCATCCAAATACTCCACACGC \\
GAPDH & GGAGCGAGATCCCTCCAAAAT & GGCTGTTGTCATACTTCTCATGG \\
\hline
\end{tabular}

RT-PCR, reverse transcription polymerase chain reaction.

concentration $\left(\mathrm{IC}_{50}\right)$ of drug-resistant cells/ $/ \mathrm{IC}_{50}$ of parental cells in the control group.

\section{Cell counting kit-8 (CCK-8) assay}

A498-DR and 786-O-DR cells were seeded into 24-well plates and divided into a sunitinib alone group and curcumin plus sunitinib group. After cells in each group were treated with corresponding drugs for $48 \mathrm{~h}, 30 \mu \mathrm{L}$ of CCK-8 reagent was added to each well, and the cells were incubated in a $5 \% \mathrm{CO}_{2}$ incubator at $37{ }^{\circ} \mathrm{C}$ for $4 \mathrm{~h}$. The absorbance was measured at $450 \mathrm{~nm}$ using a microplate reader. The drug dose-response curves were plotted, and $\mathrm{IC}_{50}$ values were calculated.

\section{Colony formation}

Cells transfected with curcumin plus sunitinib or sunitinib alone were seeded into 6-well culture plates. Three replicate wells were set up in each group, and the culture medium was replaced once every 2-3 days. After a 2-week culture, the cells were fixed in $4 \%$ paraformaldehyde solution for $30 \mathrm{~min}$ and stained with $0.1 \%$ crystal violet solution for $15 \mathrm{~min}$. The number of colonies with more than 50 cells was counted under an optical microscope.

\section{Flow cytometry}

Flow cytometry (BD FACSAria) was used to assess cell apoptosis. After the cell density was adjusted, the cells were cultured overnight. After the cells were adhered to the plates, drugs were added. After a 48-h culture, the cells were collected by centrifugation and washed twice with precooled phosphate-buffered saline (PBS). The cells were divided into a nonstaining group, annexin V-only staining group, propidium iodide (PI) staining group and PI and annexin $\mathrm{V}$ double-staining group. A total of $100 \mu \mathrm{L}$ of binding buffer was added to each group to disperse cells, and the corresponding dyes were added in the dark. Cells were incubated in the dark at room temperature for $15 \mathrm{~min}$, and then $300 \mu \mathrm{L}$ of binding buffer was added. Cells were transferred to the corresponding flow cytometry tubes for the assessment of apoptosis.

\section{Detection of iron ion content}

An Abcam Iron Assay Kit was used for detection in strict accordance with the instruction manual.

\section{RT-PCR}

RNA was extracted from target cells, and cDNA was synthesized by reverse transcription. After PCR amplification, agarose gel electrophoresis was performed. An ImageJ gel image analysis system was used to analyze the mRNA expression levels of ADAMTS18, NCOA, FTH1 and $p 53$. GAPDH was used as a reference gene. The primers of ADAMTS18, NCOA, FTH1, p53 and GAPDH could be traced in Table 1.

\section{Western blotting}

Total protein in cells was extracted using a KeyGEN Bio TECH Protein Extraction Kit (KGP1100), separated on $10 \%$ sodium dodecyl sulfate-polyacrylamide gel electrophoresis (SDS-PAGE), and transferred to nitrocellulose membranes. After blocking with skim milk powder, the blots were immunostained with primary and secondary antibodies, and the grayscale values for the bands were analyzed using a Bio-Rad imaging system. The following antibodies, with catalog numbers, were used: NCOA4 (ab86707, phosphate), FTH1 (ab65080, pH 7.4 
PBS), ADAMTS18 (orb226265, pH 7.4 PBS), and p53 (ab26, Western primary antibody dilution).

\section{Transfection with si-ADAMTS18}

Cells were transfected with $25 \mu \mathrm{L}$ of si-ADAMTS18 (orb226265, GeneChem, Shanghai, China), and si-NC was used as a control (Santa Cruz, CA, USA) at a final concentration of $40 \mathrm{nM}$. Lipofectamine 2000 (Invitrogen) was used in accordance with the manufacturer's instructions. The transfection efficiency was verified by RT-PCR.

\section{Statistical analysis}

Statistical analysis was performed using SPSS 22.0. The experimental results were analyzed using the rank sum test. Significant differences were based on a type I error $\alpha<0.05$ and type II error $\beta<0.01$.

\section{Results}

Successful establishment of sunitinib-resistant ccRCC cells

A498-DR and 786-O-DR exhibited similar changes, showing circular and dispersed growth, while A498 and 786-O cells were spindle-shaped and exhibited clustered growth (Figure 1A). Based on the MTT assay results, the $\mathrm{IC}_{50}$ values for $\mathrm{A} 498$ and $786-\mathrm{O}$ cells were $(4.68 \pm 2.33)$ and (4.82 \pm 1.62$) \mu \mathrm{M}$, respectively, and the $\mathrm{IC}_{50}$ values for A498DR and 786-O-DR cells were $(28.36 \pm 5.81)$ and $(29.42 \pm 6.37)$ $\mu \mathrm{M}$, respectively; the difference was significant $(\mathrm{P}<0.05)$ (Figure 1B). The DR indices for A498-DR and 786-ODR cells were 6.06 and 6.10, respectively. A drug-resistant cell line model was successfully established. By applying the apoptosis assay, it was discovered that sunitinib could promote A498 and 786-O apoptosis in a dose-dependent manner, yet without influencing the apoptosis of A498DR and 786-O-DR, which simultaneously verified the successfully established drug-resistant cell lines (Figure 1C).

\section{Inbibition of A498-DR and 786-O-DR cell growth by curcumin}

Curcumin plus sunitinib significantly inhibited the growth of 786-O-DR cells in a dose-dependent manner, as determined by the CCK- 8 assay (Figure $2 A$ ). The $\mathrm{IC}_{50}$ values for 786-O-DR cells were significantly reduced in the curcumin plus sunitinib group (Figure 2B). As seen in
Figure $2 C$, curcumin significantly inhibited the colony formation ability of A498-DR and 786-O-DR cells. The differences between the above groups were significant $(\mathrm{P}<0.05)$.

\section{Reversal of the resistance of ccRCC to sunitinib by curcumin through the induction of ferroptosis}

Analysis of the iron ion content in the above cell lines indicated that curcumin significantly reduced the iron content in the cell lines. The iron ion concentration in 786-O-DR treated with curcumin plus sunitinib was significantly decreased $(\mathrm{P}<0.05$, Figure $3 A)$. The mRNA and protein expression levels of ADAMTS18 and ferroptosisrelated proteins were analyzed by RT-PCR and Western blotting (Figure 3B,C,D). The results showed that curcumin plus sunitinib treatment upregulated the expression of the ADAMTS18 gene and significantly reduced NCOA4, FTH1 and $p 53$ expression levels $(\mathrm{P}<0.05)$. The results suggested that curcumin could reduce the mRNA and protein expression levels of $N C O A 4, F T H 1$, and $p 53$, indicating that curcumin may induce ferroptosis through the upregulation of the ADAMTS18 gene.

\section{Effect of curcumin on 786-O-DR activity after ADAMTS18 gene silenced}

After the transfection of 786-O-DR cells with siADAMTS18, the inhibitory effect of curcumin plus sunitinib on the proliferation of 786-O-DR was disappeared (Figure $4 A$ ). From Figure 4B,C,D, it could be found that by RT-PCR and WB assay, si-ADAMTS18 was successfully transfected into 786-O-DR cells. After silencing the ADAMTS18 gene, the difference in the expressions of p53, NCOA4 and FTH1 in each group was not significant (Figure $4 B, C, D)$. The above results suggested that after silencing the ADAMTS18 gene, the proliferation of drugresistant cell lines was restored, and the inhibitory effect of curcumin on the growth of cell lines and ferroptosis was disappeared simultaneously, indicating that the ADAMTS18 gene was critical in the process of curcumin-induced cell ferroptosis.

\section{Reversal of curcumin-induced cell death by ferroptosis inbibitors}

After incubation with $20 \mu \mathrm{M}$ ferroptosis-1 (Fer-1), a ferroptosis inhibitor, for $48 \mathrm{~h}$, the proliferation of 786-O- 

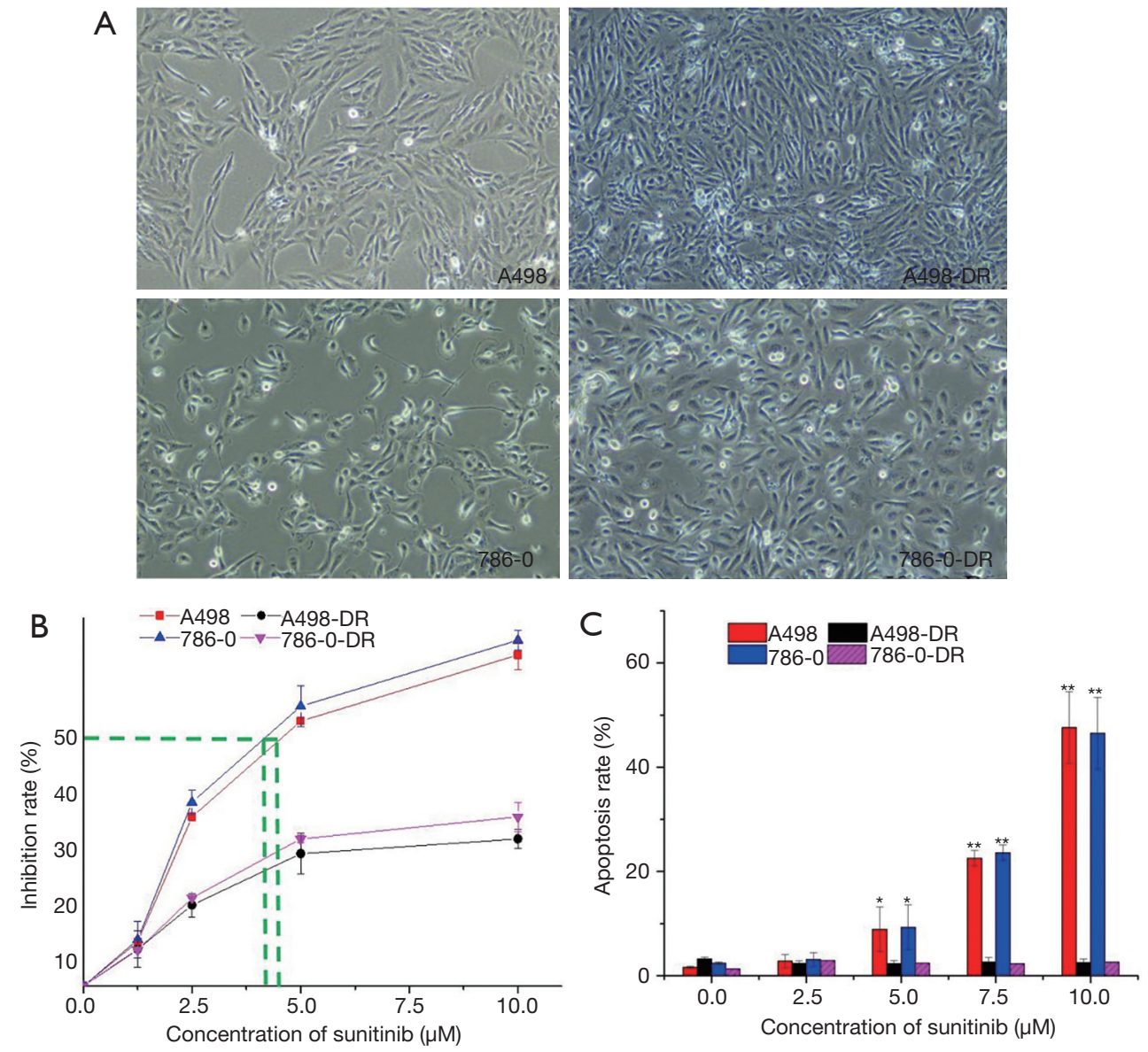

Figure 1 Successful establishment of drug-resistant ccRCC cell lines. (A) The cells of ccRCC and ccRCC-DR were observed under inverted microscope without staining, and the magnification was $10 \times 10=100$; (B) inhibition rates and $\mathrm{IC}_{50}$ values were determined by the MTT assay; (C) the apoptosis assay revealed that sunitinib promoted A498 and 786-O apoptosis in a dose-dependent manner, yet without influencing the apoptosis of A498-DR and 786-O-DR. The rank sum test was used to compare the above groups, and the difference was significant (type I error $\alpha<0.05$, and type II error $\beta<0.01)$. ${ }^{*}, \mathrm{P}<0.05 ;{ }^{* *}, \mathrm{P}<0.01$. ccRCC, clear cell renal cell carcinoma; DR, drug resistance; IC ${ }_{50}$, half maximal inhibitory concentration.

DR cells was analyzed using the CCK-8 assay. The results indicated that cell proliferation was significantly increased after Fer-1 treatment $(\mathrm{P}<0.05)$. The Fer-1 reversed the inhibitory effect of curcumin on cells, resulting in increased cell proliferation. The results also suggested that the inhibitory effect of curcumin on cell proliferation might be related to the mechanism of ferroptosis (Figure 5).

\section{Discussion}

ccRCC is a group of heterogeneous diseases originating from renal tubular epithelial cells and is a malignant tumor of the urinary system (12). Currently, the global incidence of ccRCC is relatively high. Sunitinib is a tumor-targeting drug mainly used to inhibit growth factor receptor tyrosine kinases. It is used to inhibit tumor angiogenesis. Studies have shown that sunitinib treatment can delay the progression of ccRCC by 6-7 months. However, most patients are in the stage of DR within 1 year of treatment (13), which is a clinical problem that urgently needs to be solved.

In 2015, ccRCC treatment entered the immunotherapy era. Many large-scale phase III clinical trials have been successful. Immunotherapy combined with targeted therapy or dual immunotherapy has become the first-line treatment standard for ccRCC. Based on the excellent performance of combination immunotherapy, the National Comprehensive 

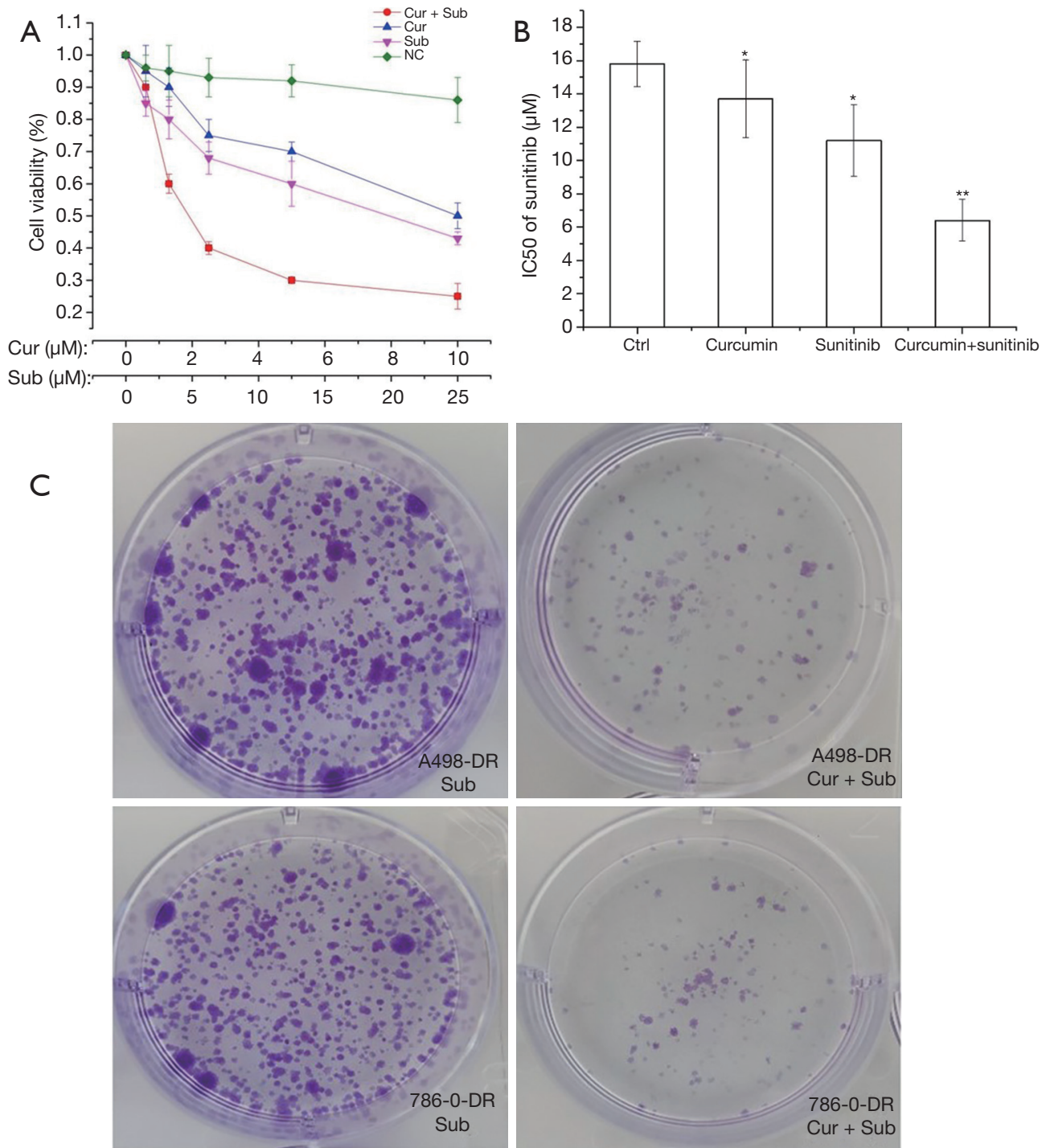

Figure 2 The inhibitory effect of curcumin on ccRCC-DR cell lines. (A) Curcumin plus sunitinib significantly inhibited the growth of 786O-DR cells in a dose-dependent manner by the CCK-8 assay; (B) the effect of curcumin and sunitinib on the $\mathrm{IC}_{50}$ value for $786-\mathrm{O}-\mathrm{DR}$ cells; (C) curcumin plus sunitinib significantly inhibited the colony forming ability of A498-DR and 786-O-DR cells. The immuno-histochemistry double staining method was used. The rank sum test was used to compare the above groups, and the difference was significant (type I error $\alpha<0.05$, and type II error $\beta<0.01)$. $\mathrm{NC}=\mathrm{ddH}_{2} \mathrm{O} .{ }^{*}, \mathrm{P}<0.05 ;{ }^{* *}, \mathrm{P}<0.01$. ccRCC, clear cell renal cell carcinoma; DR, drug resistance; IC 50 , half maximal inhibitory concentration; CCK-8, cell counting kit-8.

Cancer Network (NCCN) guidelines for kidney cancer version 1, 2021, recommend pembrolizumab combined with axitinib as the first-line preferred regimen for low-risk and intermediate-high-risk ccRCC patients and nivolumab combined with ipilimumab as the preferred first-line regimen for intermediate- and high-risk ccRCC patients. Although ccRCC treatment has entered the immunotherapy era, there are still many limitations of immunotherapy in clinical practice due to the high costs and the risk of accelerated death caused by immune hyperprogression. In addition, some drugs have not yet been marketed in most developing countries, including China.

Curcumin is a natural polyphenol extracted from the turmeric rhizome. It has antiinflammatory and antioxidant properties, activates the immune system, and can be used as an antioxidant to capture free radicals and protect living 

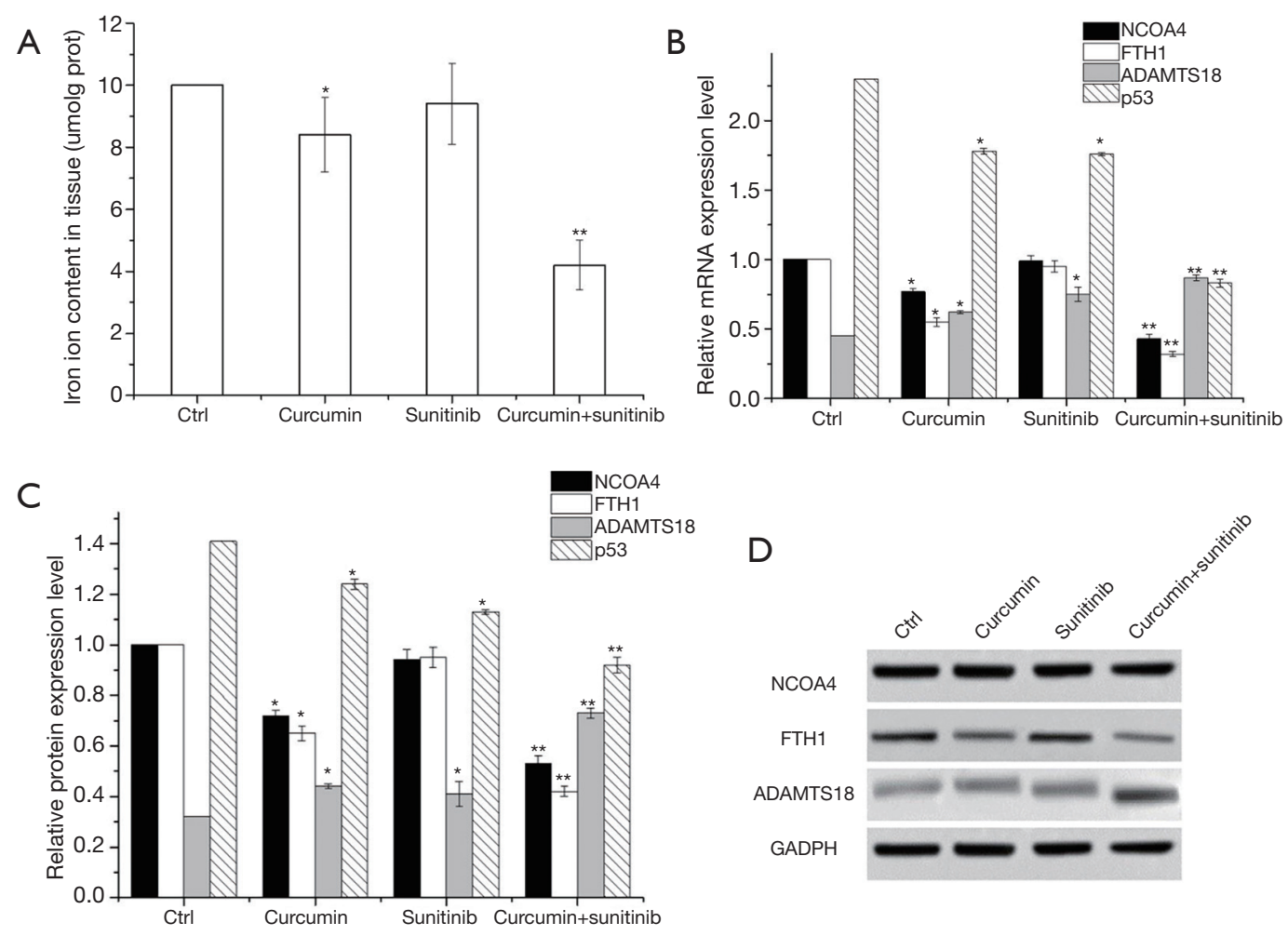

Figure 3 The ferroptosis was induced by curcumin. (A) The iron ion concentration in the different drug treatment groups; (B) RT-PCR analysis of NCOA4, FTH1, ADAMTS18 and p53 mRNA expression levels in each group; (C) Western blot analysis of NCOA4, FTH1, ADAMTS18 and $p 53$ protein expression levels in each group; (D) The specific Western blot images in original format. The rank sum test was used to compare the above groups, and the difference was significant (type I error $\alpha<0.05$, and type II error $\beta<0.01$ ). * $\mathrm{P}<0.05$; **, $\mathrm{P}<0.01$. RT-PCR, reverse transcription polymerase chain reaction.

cells (14). This study analyzed the effect of curcumin on the proliferation of sunitinib-resistant cells and found that the combination of curcumin and sunitinib reduced the proliferative activity of tumor cells. Curcumin can arrest cancer cells at different stages of the cell cycle and inhibit cancer cell proliferation by inducing apoptosis (15). Curcumin can also regulate different molecular targets and fight against human cancer. A large number of studies have also reported the inhibitory effect of curcumin on almost all types of tumor cells, such as reproductive cancers, digestive cancers, lymphoma, cancers of the immune system, urinary cancer, lung cancer, cancers of the nervous system, cancers of the skeletal system, and skin cancer. In these studies, the concentration of curcumin that yielded inhibitory activity ranged from 1 to $100 \mu \mathrm{M}$ (16).

In the study of the mechanism of cellular ferroptosis, many studies have confirmed that the cystine/glutamate reverse transporter and the glutathione peroxidase 4 pathway are closely related to cellular ferroptosis. Recently, some studies have suggested that $p 53$ and NRF2 are also involved in the mechanism of cellular ferroptosis $(17,18)$. Current studies have proposed that these pathway proteins that may be involved in cellular ferroptosis will lead to the accumulation of high levels of iron ions and reactive oxygen species in cancer cells to promote their metabolic activity and growth (19). Metabolic remodeling of cancer cells is often associated with ferroptosis sensitivity. Researchers have proposed that this phenomenon may be an adaptive response to cellular metabolic imbalance (20). Researchers have also proposed that curcumin analogs can act as ferroptosis inducers to mediate cellular ferroptosis in a dosedependent manner in the treatment of osteosarcoma (21). This study found that curcumin significantly reduced the iron ion content in drug-resistant tumor tissues, inhibited NCOA4 and FTH1 mRNA and protein expression, and enhanced sensitivity to sunitinib. Mancias et al. proposed 

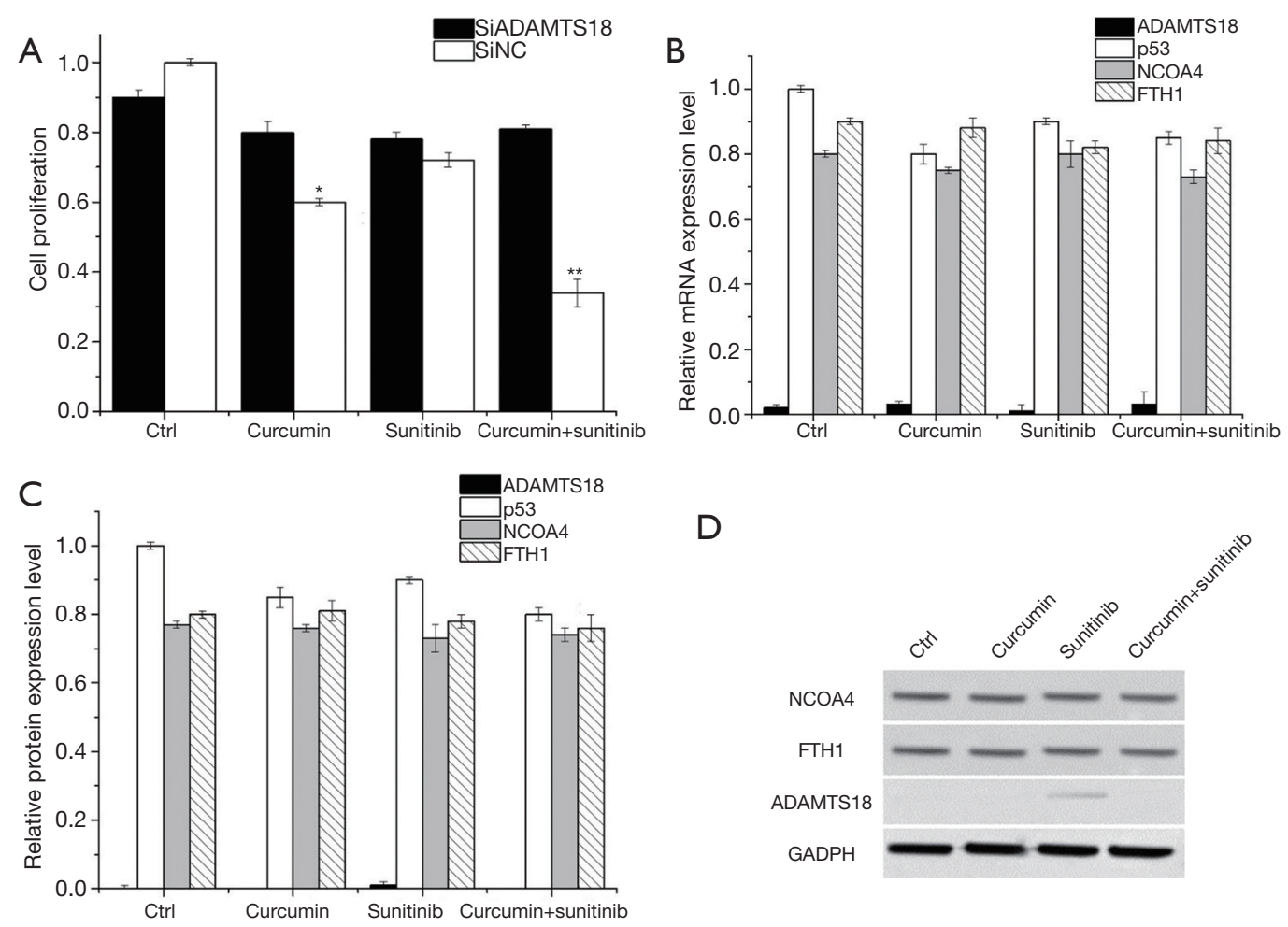

Figure 4 Effect of curcumin on the cell viability after silencing the ADAMTS18 gene. (A) Analysis of cell proliferation activity in different groups of cells after ADAMTS18 silenced; (B) analysis of NCOA4, FTH1, ADAMTS18 and p53 mRNA expression levels via RT-PCR; (C) analysis of the protein expression levels of NCOA4, FTH1, ADAMTS18 and $p 53$ in each group via Western blotting; (D) the specific Western blot images in original format. The rank sum test was used to compare the above groups, and the difference was significant (type I error $\alpha<0.05$, and type II error $\beta<0.01)$. $\mathrm{NC}=\mathrm{ddH}_{2} \mathrm{O}$. ${ }^{*}, \mathrm{P}<0.05 ;{ }^{* *}, \mathrm{P}<0.01$. RT-PCR, reverse transcription polymerase chain reaction.

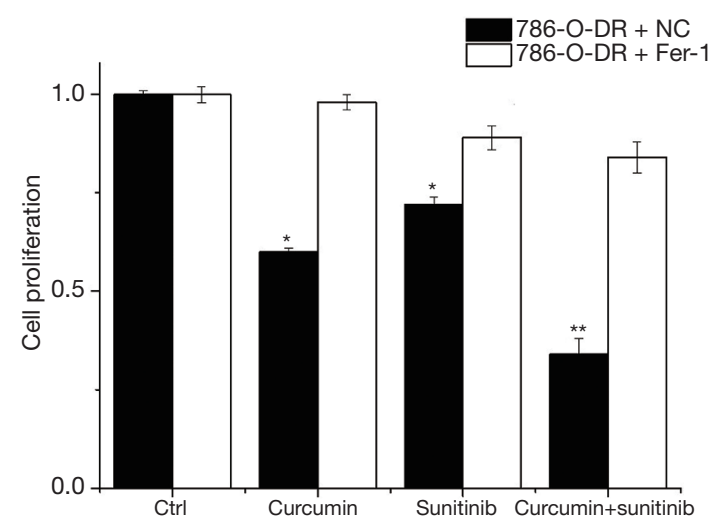

Figure 5 Reversal of curcumin-induced cell death by Fer-1. The rank sum test was used to compare the above groups, and the difference was significant (type I error $\alpha<0.05$, and type II error $\beta<0.01)$. $\mathrm{NC}=\mathrm{ddH}_{2} \mathrm{O}$. *, $\mathrm{P}<0.05 ;{ }^{* *}, \mathrm{P}<0.01$. Fer-1, ferroptosis- 1 . that NCOA4 is highly aggregated in autophagosomes and found that the delivery of ferritin to lysosomes requires NCOA4. NCOA4-deficient cells cannot degrade ferritin, resulting in a decrease in bioavailable intracellular iron (8). Studies have shown that the regulation of ferritin and iron metabolism homeostasis may become an important regulatory point for the mechanism of ferroptosis (22). Curcumin can significantly inhibit FTH1 and FTL1 gene expression in tumor tissues of nude mice (23).

In this study, after 786-O-DR cells were transfected with si-ADAMTS18, curcumin-induced ferroptosis in ccRCC was disappeared. Therefore, we propose that curcumin induces ferroptosis in tumor cells by upregulating the expression of ADAMTS18, thereby enhancing the sensitivity of ccRCC to sunitinib. Currently, no relevant studies have confirmed that ADAMTS18 is involved in the mechanism of cellular 
ferroptosis. The results of this study indicate for the first time that curcumin could mediate ferroptosis in ccRCC through ADAMTS18 gene. Unfortunately, data are limited to in vitro studies and the reported effects of curcumin still require confirmation in in vivo models in the long run.

\section{Conclusions}

In summary, this study established a sunitinib-resistant cell model and found that curcumin could reverse the sunitinib resistance in ccRCC and enhanced the sensitivity of ccRCC to sunitinib. This phenomenon may be induced through ferroptosis by upregulating ADAMTS18 gene expression.

\section{Acknowledgments}

We are particularly grateful to AJE for its language embellishment service.

Funding: This study was supported by grants from Beijing Natural Science Foundation (\#7204316) and Beijing Traditional Chinese Medicine Development Foundation (\#QN-2020-03).

\section{Footnote}

Reporting Checklist: The authors have completed the MDAR reporting checklist. Available at https://dx.doi. org/10.21037/tcr-21-227

Data Sharing Statement: Available at https://dx.doi. org/10.21037/tcr-21-227

Conflicts of Interest: All authors have completed the ICMJE uniform disclosure form (available at https://dx.doi. org/10.21037/tcr-21-227). The authors have no conflicts of interest to declare.

Ethical Statement: The authors are accountable for all aspects of the work in ensuring that questions related to the accuracy or integrity of any part of the work are appropriately investigated and resolved. The study was conducted in accordance with the Declaration of Helsinki (as revised in 2013). Institutional ethical approval and informed consent were waived.

Open Access Statement: This is an Open Access article distributed in accordance with the Creative Commons Attribution-NonCommercial-NoDerivs 4.0 International
License (CC BY-NC-ND 4.0), which permits the noncommercial replication and distribution of the article with the strict proviso that no changes or edits are made and the original work is properly cited (including links to both the formal publication through the relevant DOI and the license). See: https://creativecommons.org/licenses/by-nc-nd/4.0/.

\section{References}

1. Diaz de Leon A, Pirasteh A, Costa DN, et al. Current challenges in diagnosis and assessment of the response of locally advanced and metastatic renal cell carcinoma. Radiographics 2019;39:998-1016.

2. Adelaiye-Ogala R, Damayanti NP, Orillion AR, et al. Therapeutic targeting of sunitinib-induced AR phosphorylation in renal cell carcinoma. Cancer Res 2018;78:2886-96.

3. Pavan AR, Silva GD, Jornada DH, et al. Unraveling the anticancer effect of curcumin and resveratrol. Nutrients 2016;8:628.

4. Markowitsch SD, Schupp P, Lauckner J, et al. Artesunate inhibits growth of sunitinib-resistant renal cell carcinoma cells through cell cycle arrest and induction of ferroptosis. Cancers (Basel) 2020;12:3150.

5. Wu Y, Yu C, Luo M, et al. Ferroptosis in cancer treatment: another way to Rome. Front Oncol 2020;10:571127.

6. Lai Y, Zeng T, Liang X, et al. Cell death-related molecules and biomarkers for renal cell carcinoma targeted therapy. Cancer Cell Int 2019;19:221.

7. Qin $\mathrm{L}, \mathrm{Xu} Y, \mathrm{Xu} Y$, et al. NCOA1 promotes angiogenesis in breast tumors by simultaneously enhancing both HIF1 $\alpha$ - and AP-1-mediated VEGFa transcription. Oncotarget 2015;6:23890-904.

8. Mancias JD, Wang X, Gygi SP, et al. Quantitative proteomics identifies NCOA4 as the cargo receptor mediating ferritinophagy. Nature 2014;509:105-9.

9. Xu B, Zhang L, Luo C, et al. Hypermethylation of the 16q23.1 tumor suppressor gene ADAMTS18 in clear cell renal cell carcinoma. Int J Mol Sci 2015;16:1051-65.

10. Xu B, Peng YJ, Ma BL, et al. Aberrant methylation of the 16q23.1 tumor suppressor gene ADAMTS18 promotes tumorigenesis and progression of clear cell renal cell carcinoma. Genes Genomics 2021;43:123-31.

11. Xu B, Peng YJ, Zhu WJ. Curcumin inhibits viability of clear cell renal cell carcinoma by down-regulating ADAMTS18 gene methylation though NF- $\mathrm{K}$ B and AKT signaling pathway. Chin J Integr Med 2021. [Epub ahead of print]. doi: 10.1007/s11655-021-3445-z. 
12. Jian Y, Yang K, Sun X, et al. Current advance of immune evasion mechanisms and emerging immunotherapies in renal cell carcinoma. Front Immunol 2021;12:639636.

13. Tran TA, Leong HS, Pavia-Jimenez A, et al. Fibroblast growth factor receptor-dependent and -independent paracrine signaling by sunitinib-resistant renal cell carcinoma. Mol Cell Biol 2016;36:1836-55.

14. Rutz J, Maxeiner S, Juengel E, et al. Growth and proliferation of renal cell carcinoma cells is blocked by low curcumin concentrations combined with visible light irradiation. Int J Mol Sci 2019;20:1464.

15. Shanmugam MK, Rane G, Kanchi MM, et al. The multifaceted role of curcumin in cancer prevention and treatment. Molecules 2015;20:2728-69.

16. Heger M, van Golen RF, Broekgaarden M, et al. The molecular basis for the pharmacokinetics and pharmacodynamics of curcumin and its metabolites in relation to cancer. Pharmacol Rev 2014;66:222-307.

17. Liu J, Zhang C, Wang J, et al. The regulation of

Cite this article as: $\mathrm{Xu} \mathrm{B,} \mathrm{Zhu} \mathrm{WJ,} \mathrm{Peng} \mathrm{YJ,} \mathrm{Cheng} \mathrm{SD.}$ Curcumin reverses the sunitinib resistance in clear cell renal cell carcinoma (ccRCC) through the induction of ferroptosis via the ADAMTS18 gene. Transl Cancer Res 2021;10(7):31583167. doi: $10.21037 /$ tcr-21-227 ferroptosis by tumor suppressor p53 and its pathway. Int J Mol Sci 2020;21:8387.

18. Song X, Long D. Nrf2 and ferroptosis: a new research direction for neurodegenerative diseases. Front Neurosci 2020;14:267.

19. Battaglia AM, Chirillo R, Aversa I, et al. Ferroptosis and cancer: mitochondria meet the "Iron Maiden" cell death. Cells 2020;9:1505.

20. Chen S, Chen Y, Zhang Y, et al. Iron metabolism and ferroptosis in epilepsy. Front Neurosci 2020;14:601193.

21. Lin HY. The mechanism of curcumin analogue EF24 induces ferroptosis in osteosarcoma cells through HMOX1. Guangzhou: Southern Medical University, 2020.

22. Zuo S, Yu J, Pan H, et al. Novel insights on targeting ferroptosis in cancer therapy. Biomark Res 2020;8:50.

23. Li R, Zhang J, Zhou Y, et al. Transcriptome investigation and in vitro verification of curcumin-induced $\mathrm{HO}-1$ as a feature of ferroptosis in breast cancer cells. Oxid Med Cell Longev 2020;2020:3469840. 